\title{
Force and Motion Analysis of larval zebrafish (Danio rerio) using a body dynamics model
}

\author{
Naohisa Mukaidani \\ Department of System Cybernetics, Graduate School of Engineering, Hiroshima University, 1-4-1 Kagamiyama, \\ Higashi-Hiroshima, Hiroshima, 739-8527, Japan
}

Zu Soh

Department of System Cybernetics, Institute of Engineering, Hiroshima University, 1-4-1 Kagamiyama, HigashiHiroshima, Hiroshima, 739-8527, Japan

Shinichi Higashijima

National Institutes of Natural Sciences, Okazaki Institute for Integrative Bioscience, National Institute for Physiological Sciences, Okazaki, Aichi 444-8787, Japan

Toshio Tsuji

Department of System Cybernetics, Institute of Engineering, Hiroshima University, 1-4-1 Kagamiyama, HigashiHiroshima, Hiroshima, 739-8527, Japan

E-mail:mukaidani@bsys.hiroshima-u.ac.jp,sozu@bsys.hiroshima-u.ac.jp,shigashi@nips.ac.jp,tsuji@bsys.hiroshimau.ac.jp

www.bsys.hiroshima-u.ac.jp

\begin{abstract}
This study proposes a method of body dynamics analysis for larval zebrafish incorporating a viscoelastic body model and a fluidic environment model to support the study of development mechanisms in motion generation. The results showed that the estimated fluid drag coefficients enabled the body dynamics model to approximate the paths of actual larvae with an error level of $0.76 \pm 0.74$ [\%] to the total body length.
\end{abstract}

Keywords: zebrafish, larval zebrafish, dynamics model, drag coefficient

\section{Introduction}

Zebrafish (Danio rerio), which measure about 3-40 $[\mathrm{mm}]$ in length, have been widely used in the field of biology to study genetic diseases and the brain structure of vertebrates. Their transparency at the embryonic and larval stages of development facilitates in vivo observation of their internal organs and neural circuits, making them highly useful as a model organism for the study of phenomena observed during the stages of growth. For this reason, the behaviors of larval fish have been extensively investigated. For example, changes in swimming distance and velocity during ontogeny were systematically analyzed in the 1980s (Webb, 1984; Webb and Weihs, 1986; Fuiman and Webb, 1988). Subsequent development of computer vision techniques enabled detailed quantification of larval motion, and further analysis helped to elucidate the ontogeny of fin function (Danos and Lauder, 2008), the relationships between drag force and fin/body morphological development from the larval stage to the adult stage (McHenry and Lauder, 2006) and the effects of hydrodynamics on locomotor development (Danos, 2012). Locomotor development was also analyzed in the context of larval body waves (Müller and Leeuwen, 2004), and the function of pectoral fins at the larval 
stage was investigated by comparing the movements of wild larval fish with those of others lacking pectoral fins as a result of genetic manipulation (Green et al., 2011).

Based on the extensive body of behavioral analysis data obtained from such studies, the mechanisms supporting coordination between neural circuits and the body for appropriate motor function have recently attracted attention. For example, Budick and O'Malley classified the behavior of larvae into three repertories (Budick, 2000), and Throsen et al. analyzed fin-axis coordination (Throsen et al., 2004) to support discussion of neural control. In addition, Higashijima et al. succeed in indirect measurement of neural activities in both the motoneurons and spinal interneurons during escape behaviors using a calcium indicator called cameleon (Higashijima, 2003).

The experimental studies described above succeeded in elucidating a number of important mechanisms. The clear-cut experimental results obtained have contributed to enormous efforts regarding the control of complex experimental conditions and static analysis to eliminate the influences of individual differences in body morphology, learning ability and stages of development. However, the effects of individual differences and experimental conditions cannot be completely eliminated in experimental approaches. In addition, it is technically difficult to measure the forces produced by tiny larvae despite the importance of such information in clarifying the neural control of muscles and swimming behavior. In light of such limitations, computer simulation can be seen as a potentially effective approach. For example, Katsumata et al. constructed a body dynamics model and carried out fluid simulation for free-swimming larval zebrafish to evaluate the properties of fluid flow around them (Katsumata et al., 2009). This zebrafish larva model allowed precise description of fluid behavior.

Considering the needs of biological study, however, both fluid dynamics and local body forces generated by larvae are important in discussing the relationships between ontogeny and nerve control development. In this context, the development of a larva model that can be used to calculate forces generated from the body and those acting on the environment based on motion recorded from actual fish can be seen as effective for analyzing behavioral aspects of fish during ontogeny, such as the relationships between drag force and morphological development as analyzed by McHenry and Lauder (McHenry and Lauder, 2006). Against such a background, this paper proposes a body dynamics model incorporating the considerations of body form and viscoelasticity to support the study of developmental mechanisms in the movement of larval zebrafish. An algorithm for estimation of the external drag forces acting on such fish is also presented. The proposed model enables simulation of target phenomena in uniform virtual environments and with individual conditions, and also allows analysis for the interaction of larval zebrafish morphology and swimming movement.

\section{Body dynamics model}

As larval zebrafish measure only about $3[\mathrm{~mm}]$ in length, the forces exerted between them and their environments are difficult to measure. In this study, a body dynamics model was constructed based on the sizes and shapes of each part of these fish. A novel method of estimating drag coefficients between larval zebrafish and their environment as well as the driving torque of each joint based on dynamic analysis techniques was also introduced. Fig. 1 shows a zebrafish larva at protruding-mouth stage $(72[\mathrm{~h}])$ and a 3D morphology of the body dynamics model configured from this image. The proposed model is a rigid-link type composed of five parts: a head part consisting of one link, left and right pectoral fin parts with 10 links each, a trunk part with 10 links, and a tail fin part with 10 links. The links are interconnected by joints allowing rotation about the z-axis. The rotational motion of each link and the translational and rotational motion of a representative point $x_{\mathrm{g}}=\left[x_{g x}, \theta_{g}\right]^{T}$ of the model can be calculated from the following equation based on the Newton-Euler method:

$$
\begin{aligned}
& \mathrm{I}(\boldsymbol{q}) \ddot{\boldsymbol{q}}+\mathrm{h}(\dot{\boldsymbol{q}}, \boldsymbol{q})+\mathrm{g}(\boldsymbol{q}) \\
& \quad=\boldsymbol{\tau}-\boldsymbol{k}\left(\boldsymbol{q}-\boldsymbol{q}_{0}\right)-\mathrm{c} \dot{\boldsymbol{q}}+\sum_{r, d, j} \boldsymbol{J}_{r d j}^{\mathrm{T}} \boldsymbol{F}_{r d j} \\
& \mathrm{I}_{g}(\boldsymbol{q})\left(\ddot{\boldsymbol{x}_{g}}\right)+\mathrm{h}_{g}(\dot{\boldsymbol{q}}, \boldsymbol{q})+\mathrm{g}_{g}(\boldsymbol{q})=\sum_{r, d, j} \boldsymbol{F}_{r d j}
\end{aligned}
$$

where $\mathrm{I}(\mathrm{q})$ and $\mathrm{I}_{g}(\boldsymbol{q})$ represent the inertia matrix, $\mathrm{h}(\dot{\boldsymbol{q}}, \boldsymbol{q})$ and $\mathrm{h}_{g}(\dot{\boldsymbol{q}}, \boldsymbol{q})$ are the centrifugal force and the Coriolis force, $\operatorname{g}(\boldsymbol{q})$ and $\mathrm{g}_{g}(\boldsymbol{q})$ represent the force of gravity, $\boldsymbol{q}=\left(\boldsymbol{q}_{r j}\right) \in \mathbf{R}^{30}$ is the rotational angle vector of the joints, $\boldsymbol{q}_{0}$ is the equilibrium angle of the stiffness component at a joint, $\boldsymbol{\tau}=\left(\boldsymbol{\tau}_{\boldsymbol{r} j}\right) \in \mathbf{R}^{30}$ is a vector of rotational driving force, $\mathrm{k}$ is the stiffness coefficient 


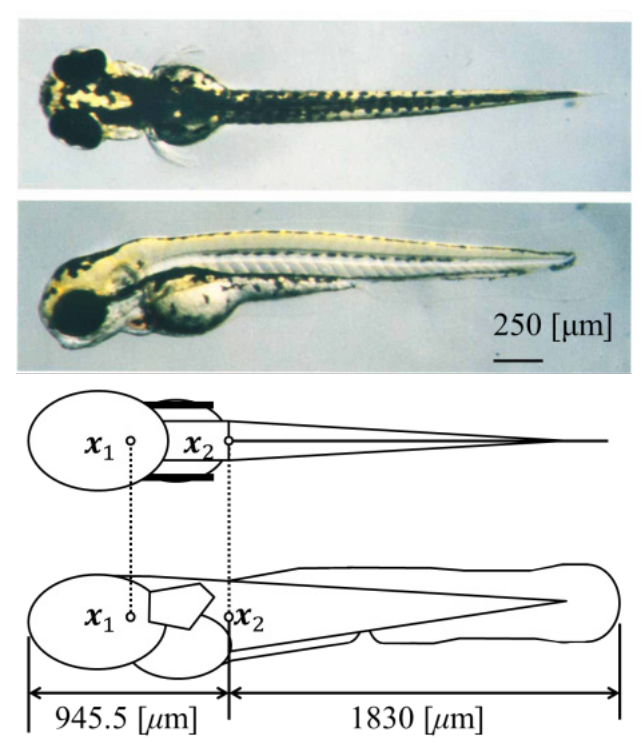

Fig. 1. Shape of a larval zebrafish and the body dynamics model. (a) shows a zebrafish larva at protruding-mouth stage (72 [h]) photographed by Kimmel et al. (Kimmel et al. 1995). Thin transparent fins surround the dorsal and ventral parts of the trunk. (b) shows the configuration of the body dynamics model approximating the image of the larva. The model's parameters were determined using this approximated shape. Position $\boldsymbol{x}_{1}$ is the centroid of the head link, and $\boldsymbol{x}_{2}$ is the joint connecting the head link and the trunk. The time traces of these two points were used to determine the drag coefficients of a fluid environment.

(Fig. 2), $c$ is the viscous damping coefficient (Fig. 2), $\boldsymbol{J}_{r d j}$ is the Jacobian matrix, and $\boldsymbol{F}_{r d j}$ is the friction force exerted between the center of gravity of a link and the external environment. In addition, the subscript $r \in(\mathrm{H}, \mathrm{B}, \mathrm{C}, \mathrm{R}, \mathrm{L})$ denotes parts of the model, with $\mathrm{H}$ representing the head, $\mathrm{B}$ the trunk, $\mathrm{C}$ the tail fin, $\mathrm{R}$ the right pectoral fin and $\mathrm{L}$ the left pectoral fin. The subscript $d \in(\mathrm{A}, \mathrm{N})$ denotes direction, with $\mathrm{A}$ representing the rostrocaudal direction of the fish and $\mathrm{N}$ the normal direction in the rostrocaudal direction. The subscript $\mathrm{j}$ denotes links sequentially numbered as $j=1,2, \cdots, 10$ from that connecting with the head part. Although the parameters on the left of the motion equation can be determined based on the measured shapes and weights of actual larval zebrafish, drag force is difficult to determine due to the size and complex morphology of the fish. In this study, the drag force $\boldsymbol{F}_{r d j}$ was approximated as a viscous resistance value proportional to the square of the velocity $\boldsymbol{v}_{r d j}$ at the

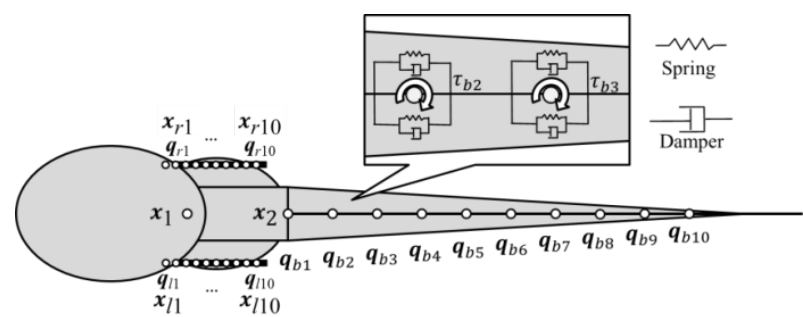

Fig. 2. Viscoelasticity of the body dynamics model. The white circles indicate inter-link joints, and $q_{r i}$ denotes the angle between the links. A pair of damper and spring units is attached to each link in parallel to represent the viscoelastic characteristics of muscles on the left and right sides. As only two-dimensional motion was considered in the model for simplification, the pairs of muscles on the dorsal and ventral parts were combined.

centroid of a link as shown in the following equation (Mochizuki and Ichikawa, 2010):

$$
\boldsymbol{F}_{r d j}=C_{r d j} \rho S_{r d j}\left\|\boldsymbol{v}_{r d j}\right\| \boldsymbol{v}_{r d j}
$$

where $C_{r d j}$ is the drag coefficient, $\rho$ is the fluid density and $S_{r d j}$ is the surface area.

As drag force significantly affects the model's motion, $C_{r d j}$ needs to be estimated. However, if different drag coefficients were assigned to each of part $r$, direction $d$ and link $j$, the number of coefficients to be estimated would be as many as 82 . Accordingly, it was assumed for simplification that the drag coefficients for the rostrocaudal direction of the pectoral fins and the tail fin $\left(C_{\mathrm{CAj}}, C_{\mathrm{RA} j}, C_{\mathrm{LAj}}\right)$ were 0 because the fins are negligibly thin compared to their total surface area. It was also assumed that the drag coefficients for the same part $r$ were identical. As a result, the friction force acting on each link can be determined via estimation of 6 drag coefficients.

\section{Drag coefficient estimation algorithm}

As Eqs. (1) and (2) describe the relationship between the motion of larval fish and related drag coefficients, these coefficients can be estimated via dynamic analysis by solving Eqs. (1) and (2). However, direct solution of drag coefficients from these equations for each time sample can yield large errors caused by video analysis noise. Accordingly, the coefficients were determined in this study by minimizing the following evaluation function for robustness: 


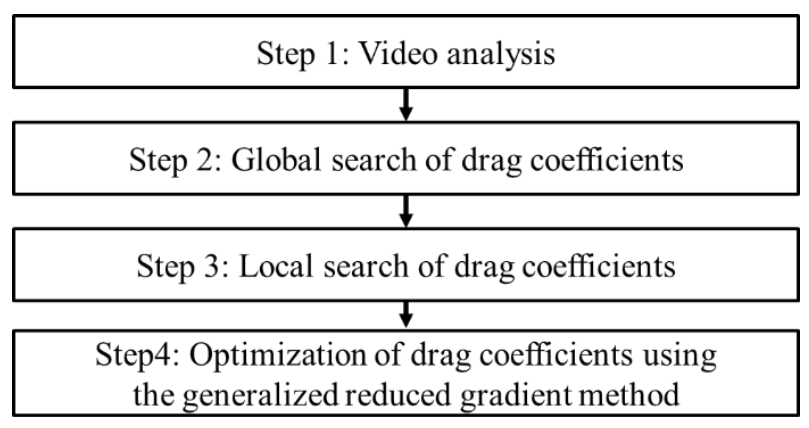

Fig. 3. Four-step flow of the algorithm used to estimate the drag coefficient of the environmental fluid. As drag coefficients are related to the paths of the body dynamics model, the proposed algorithm minimizes the difference between the paths of the actual larva and the body dynamics model. To avoid local minima, the algorithm sequentially performs global search, local search and generalized reduced gradient search where the drag coefficient sets with the smallest errors were chosen at each step.

$$
E=\sum_{t} \frac{1}{P} \sum_{p=1}^{P}\left\|x_{p}^{R}(t)-x_{p}(t)\right\|
$$

where $P$ is the number of comparison points, $x_{p}^{R}$ represents positions on an actual fish, and $x_{p}$ represents the corresponding positions on the body dynamics model as shown in Fig. 1. This evaluation function represents the error of paths between an actual fish and the model as simulated using a set of drag coefficients. The proposed algorithm minimizes this evaluation function in $M$ steps as shown in Fig. 3.

In Step 1, the movement of larval zebrafish is recorded using a high-speed camera and video image analysis is conducted (see Fig. 4). First, the tail and pectoral fins are separated as shown in Fig. 4 (a). Image processing steps including binarization, noise reduction and thinning are then performed for each frame of all the separated images to extract the centroid line of the trunk and the pectoral fins. This line is divided by points corresponding to those of the model's joints, and the time-dependent joint angles $q_{r j}$ between the divided points are calculated. As the measurement results obtained from actual fish include noise, the joint angles $q_{r j}$ are filtered using an $n$-degree low-pass Butterworth filter. Finally, the joint angles are input to the model as shown in Fig. 4 (c).

In Step 2, a global search of the drag coefficients is performed. First, a combination of drag coefficients is selected from a set $\left(C_{\mathrm{HA} 1}^{a 1}, C_{\mathrm{HN} 1}^{a 1}, C_{\mathrm{BA} 1}^{a 1}, C_{\mathrm{BN} 1}^{a 1}, C_{\mathrm{RN} 1}^{a 1}, C_{\mathrm{CN} 1}^{a 1}\right)$

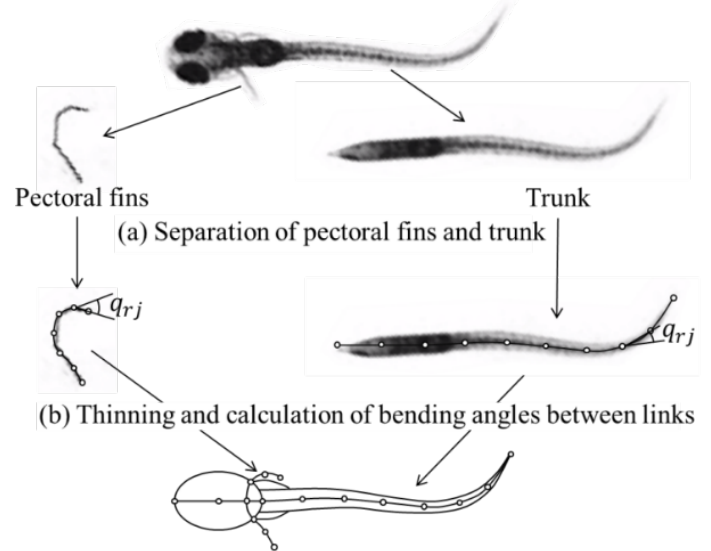

(c) Input to body dynamics model

Fig. 4. Video image analysis procedure with recording conducted at 1000 [fps]. (a) As the thinness of pectoral fins makes it difficult to perform image analysis, the trunk and fins are manually separated and lines are drawn on the blurred images of fins for interpolation. (b) Using the processed images, joint bending angles and positions are calculated using Wriggle Tracker software (Library Inc. Tokyo). (c) The outcomes of motion calculation are input to the body dynamics model, and the resulting paths are calculated based on motion equations (Eqs. (1) and (2)) .

defined using the equation below and substituted into Eq. (3).

$$
\begin{gathered}
\left(C_{\mathrm{HA} 1}^{a_{1}}, C_{\mathrm{HN} 1}^{a_{1}}, C_{\mathrm{BA} 1}^{a_{1}}, C_{\mathrm{BN} 1}^{a_{1}}, C_{\mathrm{RN} 1}^{a_{1}}, C_{\mathrm{CN} 1}^{a_{1}}\right) \\
\in\left\{N_{1} \Delta C_{\mathrm{HA} 1}^{1}, N_{2} \Delta C_{\mathrm{HN} 1}^{1}, N_{3} \Delta C_{\mathrm{BA} 1}^{1}, N_{4} \Delta C_{\mathrm{BN} 1}^{1},\right. \\
N_{5} \Delta C_{\mathrm{RN} 1}^{1}, N_{6} \Delta C_{\mathrm{CN} 1}^{1} \mid N_{1}, N_{2}, N_{3}, N_{4}, N_{5}, N_{6} \\
\left.=0,1, \cdots, N_{\mathrm{max}}^{1}\right\}
\end{gathered}
$$

where $\Delta C_{r d 1}^{1}$ is the step size for the global search yielding the $a_{1}=1,2, \cdots,\left(N_{\max }^{1}+1\right)^{6}$ variation of drag coefficient sets, and the superscript 1 indicates the step size used in the first optimization step. Data on the motion of actual larvae, including the joint angle and joint angular velocity of each link, are then substituted into Eqs. (1) and (2), and the paths are calculated by repeatedly determining the forward and inverse dynamics for each sample time. This algorithm is described below.

(i) The initial position and initial posture of a representative point on a larval zebrafish $\boldsymbol{x}_{g}(0)$ are calculated from image analysis results. Other initial values such as translational velocity $\dot{x}_{g}(0)$, joint angle $\dot{\boldsymbol{q}}(0)$, angular state velocity $\dot{\boldsymbol{q}}_{0}(0)$ and angular acceleration $\ddot{\boldsymbol{q}}_{0}(0)$ are also calculated and substituted into Eqs. (1) and (2). 
(ii) The translational velocity $\boldsymbol{v}_{r d j}(t)$ of each link is derived from the translational velocity $\dot{x_{g}}(t)$ of a representative point and the angular velocity $\dot{\boldsymbol{q}}(t)$ of each joint. The translational velocity of each link is substituted into Eq. (3) along with the selected combination of drag coefficients to determine the friction force $\boldsymbol{F}_{r d j}(t)$.

(iii) The driving torque $\boldsymbol{\tau}(t)$ and the acceleration $\ddot{\boldsymbol{x}}(t)$ are then derived from Eqs. (1) and (2) based on the substitution of $\boldsymbol{F}_{r d j}(t), \boldsymbol{q}(t), \dot{\boldsymbol{q}}(t)$ and $\ddot{\boldsymbol{q}}(t)$.

(iv) The position $\boldsymbol{x}(t)$ of the representative point on the model is derived via second-order integration for the acceleration $\ddot{\boldsymbol{x}}(t)$. By repeating steps (ii) through (iv) for each time step, the path is calculated for the selected drag coefficients. This path is then used to evaluate the coefficients by comparing it to the path of the fish using the evaluation function (Eq. (4)). After evaluation of all combinations of drag coefficients given by Eq. (5), a set of drag coefficients $\left(C_{\mathrm{HA} 1}^{b_{1}}, C_{\mathrm{HN} 1}^{b_{1}}, C_{\mathrm{BA} 1}^{b_{1}}, C_{\mathrm{BN} 1}^{b_{1}}, C_{\mathrm{RN} 1}^{b_{1}}\right.$, $C_{\mathrm{CN} 1}^{b_{1}}$ ) with smaller $d_{1}$ [\%] values for path error $\mathrm{E}$ are selected for a further search procedure in which $b_{1}=1,2, \cdots,\left(N_{\max }^{1}+1\right)^{6} d_{1} / 100$.

In Step 3, a local search is performed on the neighbors of the drag coefficient extracted in the previous step. First, a set of combinations of drag coefficients $\left(C_{\mathrm{HA} 1}^{a_{m}}, C_{\mathrm{HN} 1}^{a_{m}}, C_{\mathrm{BA} 1}^{a_{m}}, C_{\mathrm{BN} 1}^{a_{m}}, C_{\mathrm{RN} 1}^{a_{m}}, C_{\mathrm{CN} 1}^{a_{m}}\right)$ is selected. The search region for these coefficients is defined by the following equation:

$$
\begin{aligned}
& \left(C_{\mathrm{HA} 1}^{a_{2}}, C_{\mathrm{HN} 1}^{a_{2}}, C_{\mathrm{BA} 1}^{a_{2}}, C_{\mathrm{BN} 1}^{a_{2}}, C_{\mathrm{RN} 1}^{a_{2}}, C_{\mathrm{CN} 1}^{a_{2}}\right) \\
& \quad \in\left\{C_{H A 1}^{b_{1}}+N_{1} \Delta C_{H A 1}^{2}, C_{H N 1}^{b_{1}}+N_{2} \Delta C_{H N 1}^{2}, C_{B A 1}^{b_{1}}\right. \\
& \quad+N_{3} \Delta C_{B A 1}^{2}, C_{B N 1}^{b_{1}}+N_{4} \Delta C_{B N 1}^{2}, C_{R N 1}^{b_{1}} \\
& \quad+N_{5} \Delta C_{R N 1}^{2}, C_{C N 1}^{b_{1}}+N_{6} \Delta C_{C N 1}^{2} \mid N_{1}, N_{2}, N_{3}, \\
& \left.N_{4}, N_{5}, N_{6}=-N_{\max }^{m}, \cdots, 0, \cdots, N_{\max }^{m}\right\}
\end{aligned}
$$

where $\Delta C_{r d 1}^{2}$ is the step size of the drag coefficient $\left(\Delta C_{r d 1}^{2}>\Delta C_{r d 1}^{1}\right)$. The selected combination of coefficients is substituted into Eq. (3). Here, $a_{2}$ represents the number of drag coefficients in the step 2 . Dynamic analysis is performed as described in Step 2, and the simulated path is compared with that of an actual larva using the evaluation function (Eq. (4)). After evaluation of all combinations of the drag coefficients shown in Eq. (5), another set of drag coefficients $\left(C_{\mathrm{HA} 1}^{b_{2}}, C_{\mathrm{HN} 1}^{b_{2}}, C_{\mathrm{BA} 1}^{b_{2}}, C_{\mathrm{BN} 1}^{b_{2}}, C_{\mathrm{RN} 1}^{b_{2}}, C_{\mathrm{CN} 1}^{b_{2}}\right)$ with smaller $d_{2}$ [\%] values of $E$ is extracted for the end of step 3 .
In Step 4, a combination of drag coefficients $\left(C_{\mathrm{HA1}}^{\min }\right.$, $\left.C_{\mathrm{HN} 1}^{\min }, C_{\mathrm{BA} 1}^{\min }, C_{\mathrm{BN} 1}^{\min }, C_{\mathrm{RN} 1}^{\min }, C_{\mathrm{CN} 1}^{\min }\right) \quad$ with the smallest evaluation value $\mathrm{E}$ is searched using the generalized reduced gradient method (Lasdon, 1974). The initial value for this method is selected from the drag coefficients extracted in step 3 , i.e., $\left(C_{\mathrm{HA} 1}^{b_{2}}, C_{\mathrm{HN} 1}^{b_{2}}, C_{\mathrm{BA} 1}^{b_{2}}\right.$ $\left.C_{B N 1}^{b_{2}}, C_{R N 1}^{b_{2}}, C_{C N 1}^{b_{2}}\right)$.

With appropriate drag coefficients obtained using the method described above, it is possible to determine the local force generated by the fish as the torque $\boldsymbol{\tau}$ in Eq. (1) driving each link.

\section{Experiments}

To verify the efficacy of the proposed method, drag coefficient estimation was carried out using video images of swimming larvae, and the effect of pectoral fins on their swimming behavior was then explored.

\subsection{Experimental condition}

The experimental conditions configured for the drag coefficient estimation algorithm (Fig. 3) are shown below.

\subsubsection{Video image analysis conditions}

- Frame rate: $f_{s}=1000$ [fps]

- Measurement duration: $\mathrm{t}_{\mathrm{m}}=0.27[\mathrm{~s}]$

- Low-pass filter: $f_{\text {low }}=70[\mathrm{~Hz}], \mathrm{n}=2$

- The pectoral fin images used in the experiment were manually interpolated for every frame due to an insufficient level of clarity for analysis. In addition, the pectoral fins and the trunk were separated via manual processing by painting unrelated parts of each frame in white as a background color (see Fig. 4 (b)).

- Software used for video image analysis: Wriggle Tracker (Library Inc., Tokyo)

\subsubsection{Configuration for drag coefficient estimation}

$$
\begin{gathered}
\text { - Step size: } \Delta \mathrm{C}_{\mathrm{HA} 1}^{1}=1.3 \times 10^{-8}, \\
\Delta C_{\mathrm{HN} 1}^{1}=6.3 \times 10^{-7}, \Delta C_{\mathrm{BA} 1}^{1}=1.3 \times 10^{-7}, \\
\Delta C_{\mathrm{BN} 1}^{1}=1.3 \times 10^{-4}, \Delta C_{\mathrm{RN} 1}^{1}=1.3 \times 10^{-2}, \\
\Delta C_{\mathrm{CN} 1}^{1}=1.3 \times 10^{-4}, \Delta C_{\mathrm{HA} 1}^{2}=1.3 \times 10^{-9}, \\
\Delta C_{\mathrm{HN} 1}^{2}=0.63, \Delta C_{\mathrm{BA} 1}^{2}=1.3 \times 10^{-8}, \\
\Delta C_{\mathrm{BN} 1}^{2}=1.3 \times 10^{-5}, \Delta C_{\mathrm{RN} 1}^{2}=1.3 \times 10^{-3}, \\
\Delta C_{\mathrm{CN} 1}^{2}=1.3 \times 10^{-5}
\end{gathered}
$$

- Number of searches: $N_{\max }^{1}=100, N_{\max }^{2}=4$ 


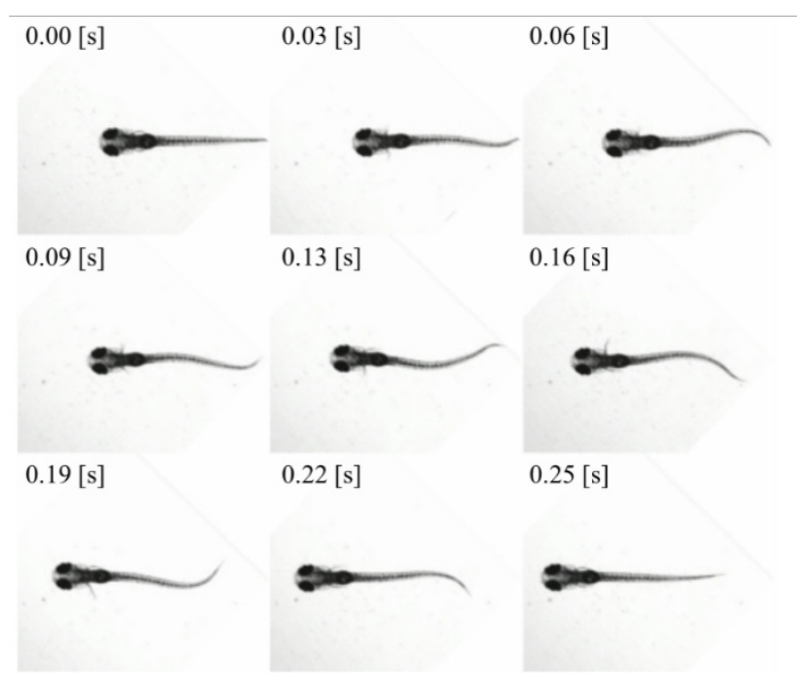

Fig. 5. Video images of wild larval zebrafish motion. The pictures show temporal changes in posture and position for every 0.03 seconds from when the larva started swimming until it stopped.

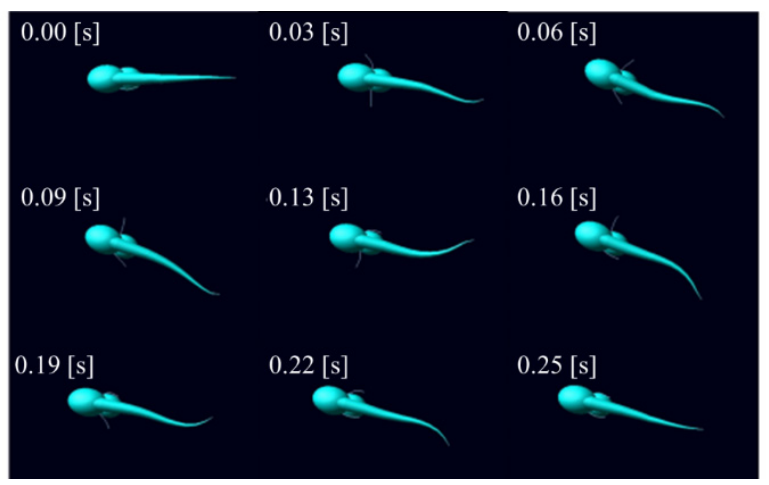

Fig. 6. Video images of the body dynamics model before optimization. The pictures demonstrate a typical example with randomly chosen drag coefficients in which drag force precluded body thrust.

\subsubsection{Dynamic analysis conditions}

- Equilibrium angle (Eq. 1): $q_{0}=0$

- Density of water (Eq. 3): $\rho=1.00\left[\mathrm{~g} / \mathrm{cm}^{3}\right]$

(at a water temperature of $20\left[{ }^{\circ} \mathrm{C}\right]$ )

- Larval model length: $l_{b}=2.78[\mathrm{~mm}]$

- Mass density of larval model: 3.33 [g/ $\left.\mathrm{cm}^{3}\right]$

(Calculated based on reference of Avella et al. (2012))

- Form of link sets: as shown in Figs. 1 and 4

- Joint stiffness: $15.5\left[\mathrm{kNm} \cdot \mathrm{rad} / \mathrm{m}^{2}\right]$ (Long, 1998)

- Joint viscosity: 0.84 [kNms $\left.\cdot \mathrm{rad} / \mathrm{m}^{2}\right]$ (Long, 1998)

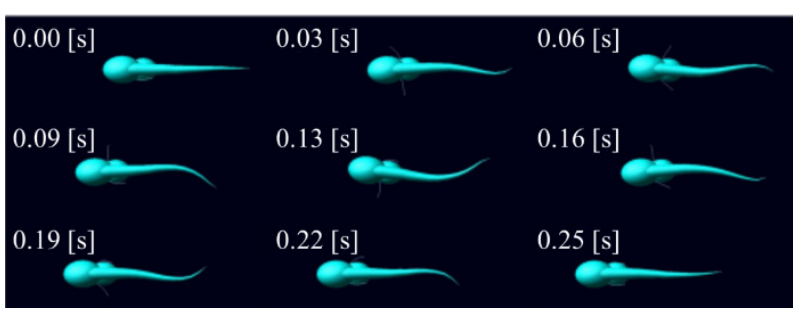

Fig. 7. Video images of the body dynamics model after optimization. When optimal drag coefficients were chosen, the model accurately traced the paths observed from an actual

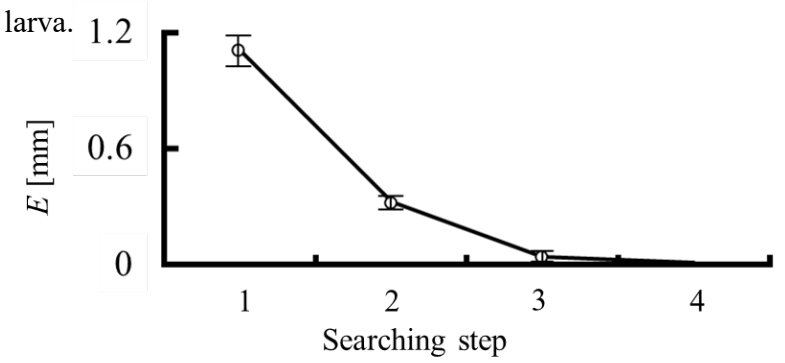

Fig. 8. Video images of the body dynamics model after optimization. When optimal drag coefficients were chosen, the model accurately traced the paths observed from an actual larva

\subsubsection{Configuration reading evaluation function}

- Number of comparison points in evaluation function (Eq. 4): $N=2$

- Position of fish for evaluation (Eq. 4):

- Centroid of head $(i=1)$,

- Point of joint connecting head and trunk $(i=2)$ (see Fig. 1)

\subsection{Drag coefficient estimation}

Drag coefficients were estimated based on the algorithm presented in Section 2.1. Fig. 5 shows video images of the wild larval zebrafish. Fig. 6 shows the simulated movement of the body dynamics model before optimization, and Fig. 7 shows that after. Fig. 8 shows the average path error between a wild fish and the body dynamics model as given by Eq. (4), and indicates a gradual decrease in each step. After drag coefficient optimization using the generalized reduced gradient method, the path error approached 0 at about $0.76 \pm 0.74$ [\%] compared to the body length. Fig. 9 shows the path of larval movement, with (a) and (b) indicating a position of $70[\%]$ normalized by body length with the tip of the head as $0[\%]$ and the end of the tail as 100 


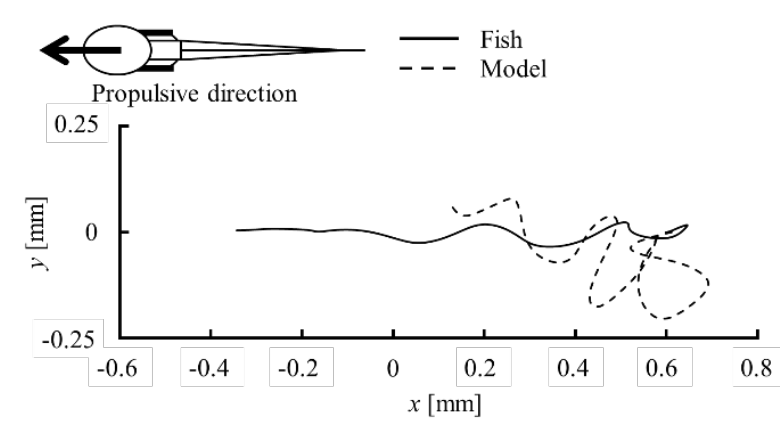

(a) Paths of the fish and the pre-optimization model

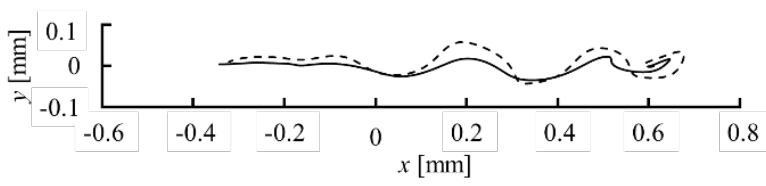

(b) Paths of the fish and the post-optimization model

Fig. 9. Comparison of paths obtained before and after optimization. (a) shows a typical path of the body dynamics model when drag coefficients were chosen randomly, and corresponds to the images shown in Fig. 6. (b) demonstrates the path of the body dynamics model given optimal drag coefficients, and corresponds to the images shown in Fig. 7. It is particularly notable that the model successfully traced the backward swimming observed when the actual fish began swimming.

[\%]. The solid line shows the path of the larval zebrafish, and the dashed line shows the path of the model before and after drag coefficient optimization. Comparison of the optimized model and the larval zebrafish shows that the former captured the characteristics of fish motion in which both the model and the larval zebrafish started by swimming backward then forward. These results indicate that the body dynamics model with drag coefficients estimated using the proposed algorithm successfully reproduces the movement of wild larval zebrafish.

\section{Results and Discussion}

Although the pectoral fins of adult zebrafish are known to contribute to thrust (Webb, 1973), their function in developmental-stage larval zebrafish has not been fully elucidated. In this study, the influence of pectoral fins on swimming was examined by comparing the resultant force generated from the pectoral fin part with those of other parts. These resultant forces were calculated by simply adding all force vectors acting on the corresponding links via the following equation:

$$
\boldsymbol{F}_{r}^{\mathrm{res}}=\sum_{d j} \boldsymbol{F}_{r d j}
$$

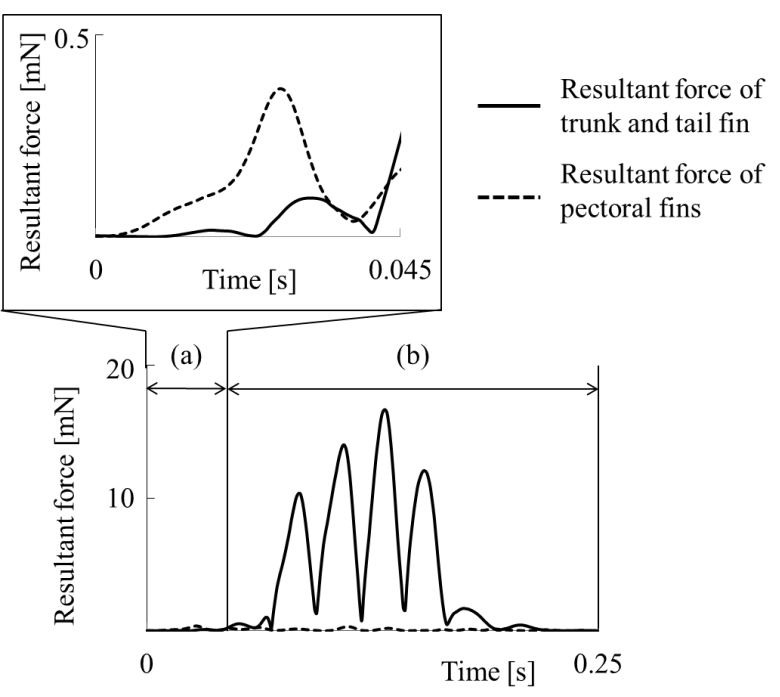

Fig. 10. Resultant force of the trunk and fins. The broken line shows the resultant force of the pectoral fin part, and the solid line shows that of the trunk and tail part. Overall, the force generated from the pectoral fins is negligible compared to that generated from the trunk, which is consistent with biological experiment results [Green et al. 2011]. However, in period (a), the larvae initiated swimming and the model predicted that the pectoral fins would generate force to push the fish backward.

The resultant force generated by the trunk and tail fin part $\left(\boldsymbol{F}_{\mathrm{BC}}^{\text {res }}=\boldsymbol{F}_{\mathrm{B}}^{\text {res }}+\boldsymbol{F}_{\mathrm{C}}^{\text {res }}\right)$ and that of the pectoral fin part $\left(\boldsymbol{F}_{\mathrm{RL}}^{\text {res }}=\boldsymbol{F}_{\mathrm{R}}^{\text {res }}+\boldsymbol{F}_{\mathrm{L}}^{\text {res }}\right)$ were calculated. In Fig. 10, period (a) shows the time immediately after the larva started swimming $(0-0.045[\mathrm{~s}])$ during which it moved backward, and period (b) shows the duration of forward swimming $(0.045-0.25[\mathrm{~s}])$. For period (b), the analysis results showed that the average resultant force of the trunk and tail fin part was about $\overline{\boldsymbol{F}}_{\mathrm{RL}}^{\text {res }}=78[\mu \mathrm{N}]$ and that of the pectoral fin part was about $\overline{\boldsymbol{F}}_{\mathrm{BC}}^{\text {res }}=4068$ $[\mu \mathrm{N}]$. As the resultant force of the pectoral fin part accounts for only $2[\%]$ of the total force generated by the body dynamics model, its influence on swimming can be considered negligible. This result is consistent with those of the experimental studies. Green et al. assumed that pectoral fins affected the movement of larval zebrafish and conducted motion analysis on actual fish (Green et al., 2011). The results showed a minimal influence from pectoral fins on swimming in the larval stage; rather, the fins are used to supply oxygen to the gills at this stage of development. This outcome verified the efficacy of the proposed model and drag coefficient estimation algorithm, indicating their 
suitability for the prediction of unknown dynamic characteristics. In this context, focus was subsequently placed on period (a), for which the average resultant force of the trunk and tail fin part was about $\overline{\boldsymbol{F}}_{\mathrm{BC}}^{\text {res }}=39$ $[\mu \mathrm{N}]$ and that of the pectoral fin part was about $\overline{\boldsymbol{F}}_{\mathrm{RL}}^{\mathrm{res}}=121[\mu \mathrm{N}]$. These results indicate that the trunk and tail fin part have a smaller influence in period (a), allowing pectoral fin movement to contribute more to swimming. Indeed, the fish was observed to maneuver its pectoral fins toward its head, thereby creating force for backward swimming. Such pectoral fin movement may thus be applied to generate force in order to determine the direction of swimming. In this way, the proposed model can provide guidelines for biological experiments.

\section{Conclusions and future work}

This paper proposed a body dynamics model for larval zebrafish and reported on dynamic analysis of the species. The measured paths of wild larval fish were compared with those obtained from swimming simulation using the proposed model. The results indicated that the model can be used to approximate paths with a high accuracy level of $0.76 \pm 0.74$ [\%] in relation to body length. It was also found to be capable of reproducing paths with characteristics similar to those of wild larval fish as well as supporting estimation of how pectoral fins affect swimming.

In future work, the author plans to elucidate the swimming mechanism of larval fish with focus on the motion generation mechanisms hidden behind the neural circuit. To this end, muscle and neural circuit models will be constructed, and the mechanisms behind the development of swimming behavior in larval zebrafish will be analyzed

\section{Reference}

1. M. A. Avella, A. Place, S.-J. Du, E. Williams, S. Silvi, Y. Zohar and O. Carnevali, (2012). Lactobacillus rhamnosus accelerates zebrafish backbone calcification and gonadal differentiation through effects on the GnRH and IGF systems, PLoS One 7, (2012), e45572

2. S. Budick and D. O'Malley, Locomotor repertoire of the larval zebrafish: swimming, turning, and prey capture, $J$. Exp. Biol. (2000), 203, 2565-2579.
3. N. Danos and G. V. Lauder, The ontogeny of fin function during routine turns in zebrafish Danio rerio, J. Exp. Biol. 210, (2008), 3374-3386.

4. L. Fuiman and P. W. Webb, Ontogeny of routine swimming activity and performance in zebra danios (Teleostei: Cyprinidae), Anim. Behav. (1988), 36, 250261.

5. M. H. Green, R. K. Ho and M. E. Hale, Movement and function of the pectoral fins of the larval zebrafish (Danio rerio) during slow swimming, Exp. Bio. 214, (2011), 3111-3123.

6. S. Higashijima, Y. Hotta and H. Okamoto, Visualization of cranial motor neurons in live transgenic zebrafish expressing green fluorescent protein under the control of the islet-1 promoter/enhancer, J. Neurosci, 20, (2000), 206-18.

7. Y. Katsumata, U. K. Muller and H. Liu, (2009). Computation of Self-Propelled Swimming in Larva Fishes, Bio. Sci. and Eng. 4, 1, 54-66.

8. C. B. Kimmel, W. W. Ballard, S. R. Kimmel, B. Ullmann and T. F. Schilling, Stages of Embryonic Development of the Zebrafish, Dev. Dynam. 203, (1995), 253-310.

9. L. S. Lasdon, R. L. Fox and M. W. Ranter, Nonlinear optimization using the generalized reduced gradient method, Revue Francaise d'Automatique. Informatique et Recherche Operationelle. 3, (1974), 73-104.

10. J. H. Long Jr. Muscles, Elastic Energy, and the Dynamics of Body Stiffness in Swimming Eels, Amer. Zool. 38, (1998), 771-792.

11. M. McHenry and G. Lauder, Ontogeny of form and function: locomotor morphology and drag in zebrafish (Danio rerio), J. Morphol. 267, (2006), 1099-1109.

12. O. Mochizuki and S. Ichikawa, Hydrodynamics for learning from creatures (in Japanese), Yokendo, (2010).

13. U. K. Müller and J. L. van Leeuwen, Swimming of larval zebrafish: ontogeny of body waves and implications for locomotory development, J. Exp. Biol. 203, (2004), 853 868.

14. D. H. Thorsen, J. J. Cassidy and M. E. Hale, Swimming of larval zebrafish: fin-axis coordination and implication for function and neural control, J. Exp. Biol. 207, (2004), 4175-4183.

15. P. W. Webb, Effects of partial caudal-fin amputation on the kinematics and metabolic rate of underyearling sockeye salmon (Oncorhynchus nerka) at steady swimming speeds, J. exp. Biol. 59, (1973), 565-581.

16. P. W. Webb, Form and function in fish swimming. Scient. Am., 251, (1984), 72-82.

17. P. W. Webb and D. Weihs, Functional locomotor morphology of early life history stages of fishes, Trans. Am. Fish. Soc. 115, (1986), 115-127. 